\title{
Disease Progression, CTCAE
}

National Cancer Institute

\section{Source}

National Cancer Institute. Disease Progression, CTCAE. NCI Thesaurus. Code C143201.

Death due to disease progression that cannot be attributed to a CTCAE term associated with Grade 5. 